\title{
The FRaNKIE code: a Tool for Calculating Multi-Wavelength Interstellar Emissions in Galaxies
}

\author{
Troy A. Porter ${ }^{* a}$, G. Jóhannesson ${ }^{b}$, and I. V. Moskalenko ${ }^{a}$ \\ ${ }^{a}$ Hansen Experimental Physics Laboratory and Kavli Institute for Particle Astrophysics and \\ Cosmology, Stanford University, Stanford, CA 94305, U.S.A. \\ ${ }^{b}$ Science Institute, University of Iceland, Dunhaga 5, IS-107 Reykjavík, Iceland \\ E-mail: tporterestanford.edu, gudlaugueglast2. stanford.edu, \\ imosestanford.edu
}

The Fast Radiation Numerical Kalculation for Interstellar Emission (FRaNKIE) code is a Monte Carlo modelling tool for calculating electromagnetic emissions from galaxies. The code is highly parallel and optimised for both CPUs and co-processor accelerators. FRaNKIE takes into account the interaction of the photon field with the interstellar medium in a self-consistent way, providing a detailed model for the intensity and angular distribution of electromagnetic emission throughout the modelling volume. Implementation details of the code are described and the results of its application to calculate the 3D interstellar radiation field (ISRF) of the Milky Way are presented. The ISRF is an essential input to cosmic ray propagation codes for calculating the energy losses by leptons from inverse Compton scattering, and the resulting diffuse $\gamma$-ray emission.

The 34th International Cosmic Ray Conference,

30 July- 6 August, 2015

The Hague, The Netherlands

${ }^{*}$ Speaker. 


\section{Introduction}

The Milky Way interstellar radiation field (ISRF) is the result of emission by stars, and the scattering, absorption, and re-emission of absorbed starlight by dust in the interstellar medium (ISM). Accurate modelling for the ISRF is essential for understanding the interstellar emission produced by $\mathrm{CRs}$ from $\mathrm{keV}$ to $\mathrm{GeV}$ energies [1, 2, 3], $\gamma$-ray emission from supernova remnants (e.g., [4]), pair-production via $\gamma$-ray absorption on the ISRF [5], the Extended Red Emission in the interstellar medium [6], the determination of the isotropic component of the extragalactic $\gamma$-ray background [7, 8], and many other studies.

The first calculation considering the broadband (optical to far-IR) spectral energy distribution (SED) as a function of Galactocentric distance was made in [9]. Subsequent works [10,11] employed models of increasing complexity, but the crucial coupling of dust absorption of starlight with re-emission was not included. The fully self-consistent radiation transport calculation treating the absorption and re-emission of starlight by dust was done in [12] using the first version of the Fast Radiation Numerical Kalculation for Interstellar Emission (FRaNKIE) code, which used a ray tracing method. The code was extended in [1] (version 2) to calculate the full angular distribution of the intensity of the ISRF from ultraviolet to far-IR wavelengths, which is essential for the calculation of the anisotropic IC emission [13].

In this contribution the latest version of the FRANKIE code is described, which now uses a parallel Monte Carlo method for photon propagation. FRaNKIE is written in C++ and available as a standalone executable as well as a library. The latter facility allows it to be directly coupled to other codes. Its high degree of configurability allows the code to be applied to physical problems other than those that it was originally conceived to address.

\section{The FRaNKIE code}

All of the components of a "run" of the FRaNKIE code are controlled by the user via a set of of xml files that specify the simulation geometry, stellar luminosity and ISM density distributions, and other relevant parameters. The spatial distribution for the stellar luminosity can be discrete (individual stars) or continuous (a density averaged over a volume element). Pre-coded primitives (e.g., exponential disk, triaxial bulge, spiral arms) are available for the latter case, which can be combined to produce composite spatial distributions of considerable complexity. For discrete and continuous distributions the user can also provide the spatial model in tabulated form. The frequency dependence of the stellar luminosity for each component of the spatial distribution (stars or stellar densities) can be selected from pre-defined tables including the usual stellar types (Main Sequence stars, Giants, etc.), or supplied by the user. The ISM densities and their frequency dependent properties, such as the scattering/absorption cross sections of dust particles, are also specified for a run using pre-defined data, or user-supplied in tabular form.

The Monte Carlo method is similar to those described in $[14,15,16]$. The system volume is segmented into cells with the number of luminosity packets injected per cell determined according to the ratio of the cell stellar luminosity to the system stellar luminosity for a given number of total injected particles $N_{\text {total }}$. The parallelisation is done using OpenMP ${ }^{18}$ with one thread per cell,

\footnotetext{
${ }^{18} \mathrm{http}: / /$ openmp.org/wp/
} 
injecting all particles for the cell. Each packet emitted within a cell is released with an isotropic angular distribution uniformly over the cell with frequency sampled from the stellar luminosity spectrum at that location.

Following emission, the first interaction is forced to increase sampling efficiency [14]. The interaction length is sampled according to the dust optical depth in the emitted direction, and the photon is propagated the corresponding distance. The fractional path-length intersecting all cells between the initial and final positions is recorded, which is used for the grain stochastic heating calculation (see below) and to determine the radiation pressure for each cell [17, 18]. The interaction is either a scattering or absorption (or the packet is lost from the system if the interaction length is outside the boundary). The probability for a scattering or absorption depends on the frequency dependence of the scattering and absorption cross section for the user-specified grain mixture. If the packet is scattered, the new direction is calculated according to the dust grain type (determined by the relative sizes of the scattering cross sections for the assumed grain mixture) scattering matrix. If the packet is absorbed, the absorbing grain type is determined from the relative sizes of the absorption cross sections for the assumed mixture. If the absorbing grain type is "large", that is, it reemits in thermal equilibrium, the "temperature correction" method [19] is used. To enforce radiative equilibrium the dust immediately reemits a luminosity packet, where the reemitted frequency is chosen from a distribution that corrects the temperature of the dust prior to its new temperature following the absorption of the original packet. If the absorbing grain type undergoes stochastic heating (e.g., the nanograin components of the dust mixture) it cannot be treated using this method, and so the amount of luminosity absorbed in the cell is recorded and the packet is removed from the system (to be dealt with in a subsequent step, as described below). The scattered or absorbed/reemitted packets propagate in the system until they either escape, or are fully absorbed. This process is then repeated for each injected luminosity packet.

To treat the packets absorbed on the grains undergoing stochastic heating, the frequencydependent absorbed luminosity (recorded earlier) by these grains is used to compute the stochastic heating emissivity for each cell using the "thermal continuous" method [20]. This is parallelised using accelerator co-processors [21]. The procedure used above for injection of stellar photons is then followed, except the stochastic heating emissivity distribution is used in place of the stellar luminosity distribution. With this method energy conservation better than $\sim 10^{-4}$ is achieved after a single pass.

The frequency-dependent intensity is recorded as HEALPix images [22] at user-specified locations, with the (frequency-dependent) number density obtained by simply integrating over $4 \pi$ sr. A full range of photometric filters (UVB, DIRBE, Spitzer IRAC/MIPS, etc.) are encoded so that the intensity is available with the raw frequency dependence, or convolved with the filters to allow for direct comparison with data; again, this is fully user-specified via the xml configuration files.

\section{The Interstellar Radiation Field of the Milky Way in 3D}

The ISRF model ${ }^{1}$ used for essentially all calculations of the IC emission from the Milky Way is based on a cylindrically symmetric 2D calculation using the FRaNKIE code [1]. However, this

\footnotetext{
${ }^{1}$ All data files related to ISRF models calculated with the FRaNKIE code are available from http://galprop.stanford.edu.
} 
is an approximation made due to limitations of earlier versions of the code. Here, the parameters used to generate the 2D ISRF used in a recent work [3] are employed for a full 3D calculation (that is, the averaging over azimuthal angle is not used). In addition, a model for the spiral arms for the stellar luminosity distribution and dust/gas is used. The non-axisymmetric stellar bulge/bar and arms are important for predicting the observed intensity at near- and far-IR frequencies where these features are clearly visible, e.g., [23] and see below.

The model follows $[24,25]$ using a table of stellar spectral types comprising main sequence stars, giants, and exotics to represent the luminosity function (LF) for each of the spatial components. The spectral templates for each stellar type are taken from the semi-empirical library given in [26]. The normalisations per stellar type are obtained by adjusting the space densities to reproduce the observed LFs in the V-and K-band for the thin disc. The LFs for the other spatial components are obtained by adjusting weights per component for each of the stellar types relative to the normalisations obtained for the thin disc LF. The spatial densities of the thin and thick disc are modelled as exponential disks. For the thin disc available estimates for the radial scale length range from $\sim 2 \mathrm{kpc}$ to $\sim 4 \mathrm{kpc}$ while for the thick disc estimates give the range $\sim 3-4 \mathrm{kpc}$, e.g., [27, 28, 29, 30, 31]. Radial scale lengths of $2.5 \mathrm{kpc}$ and $3.5 \mathrm{kpc}$ for the thin and thick disc, respectively, are used in the present work. The thin disc has a hole interior of a Galactocentric radius of $\sim 1.7 \mathrm{kpc}$, following [23]. The scale height of the stellar classes in the thin disc follows [25], while the thick disc is characterised by a single scale height of $0.75 \mathrm{kpc}$, which is approximately the middle of values from the literature, e.g., [30]. The local thick disc to thin disc normalisation, $\rho_{\text {thick }}\left(R_{\odot}\right) / \rho_{\text {thin }}\left(R_{\odot}\right)$, is assumed to be $5 \%$. The stellar arms are taken from [32] normalised to the local thin disc LF. The stellar halo is an oblate symmetrical spheroid with axial ratio $c / a=0.7$ and power-law density profile $\rho_{\text {halo }} \propto r^{-2.8}$, intermediate between values found from Sloan data $[29,30]$. The local halo to thin disc normalisation, $\rho_{\text {halo }}\left(R_{\odot}\right) / \rho_{\text {thin }}\left(R_{\odot}\right)$, is $0.5 \%$. The bulge is assumed to be "boxy" following [33] with geometrical parameters taken from their paper. The bulge input luminosity is normalised to the K-band luminosity given in [23] for an assumed $R_{\odot}=8.5 \mathrm{kpc}$.

The dust model includes graphite, polycyclic aromatic hydrocarbons (PAHs), and silicate grains. The grains are assumed spherical and electrically neutral ${ }^{2}$, with the absorption and scattering efficiencies based on the updated interstellar dust model described in [34]. The dust grain abundance and size distribution are taken from [35] (their best-fit Galactic model). Dust follows the Galactic gas distribution uniformly mixed with the ISM [36]; here we use a two component model comprised of an exponential disk and 4 spiral arms, with the spiral arms as specified for the stellar luminosity model above. The dust-to-gas ratio scales with the Galactic metallicity gradient. Estimates for the Galactic [O/H] gradient vary in the range $0.04-0.07 \mathrm{dex} \mathrm{kpc}^{-1}$ [37]; a value of 0.07 is used here. The variation of the metallicity gradient influences the scattering and redistribution of the mainly UV and blue component of the ISRF into the infrared: increased metallicity implies more dust, and therefore increased scattering and absorption of the star light.

Figure 1 compares the local intensity of the ISRF calculated for the above model for the DIRBE band 2 and band 9 filters (central wavelengths 2.2 and $148 \mu \mathrm{m}$, respectively) with the data retrieved from the NASA HEASARC archive ${ }^{3}$. The agreement for the near-IR filter is good

\footnotetext{
${ }^{2}$ The code can treat more complicated grain geometries, e.g., ellipsoidal or cylindrical, as well as charged grain mixtures. The corresponding absorption and scattering cross sections need only be supplied.

${ }^{3}$ http://lambda.gsfc.nasa.gov/
} 

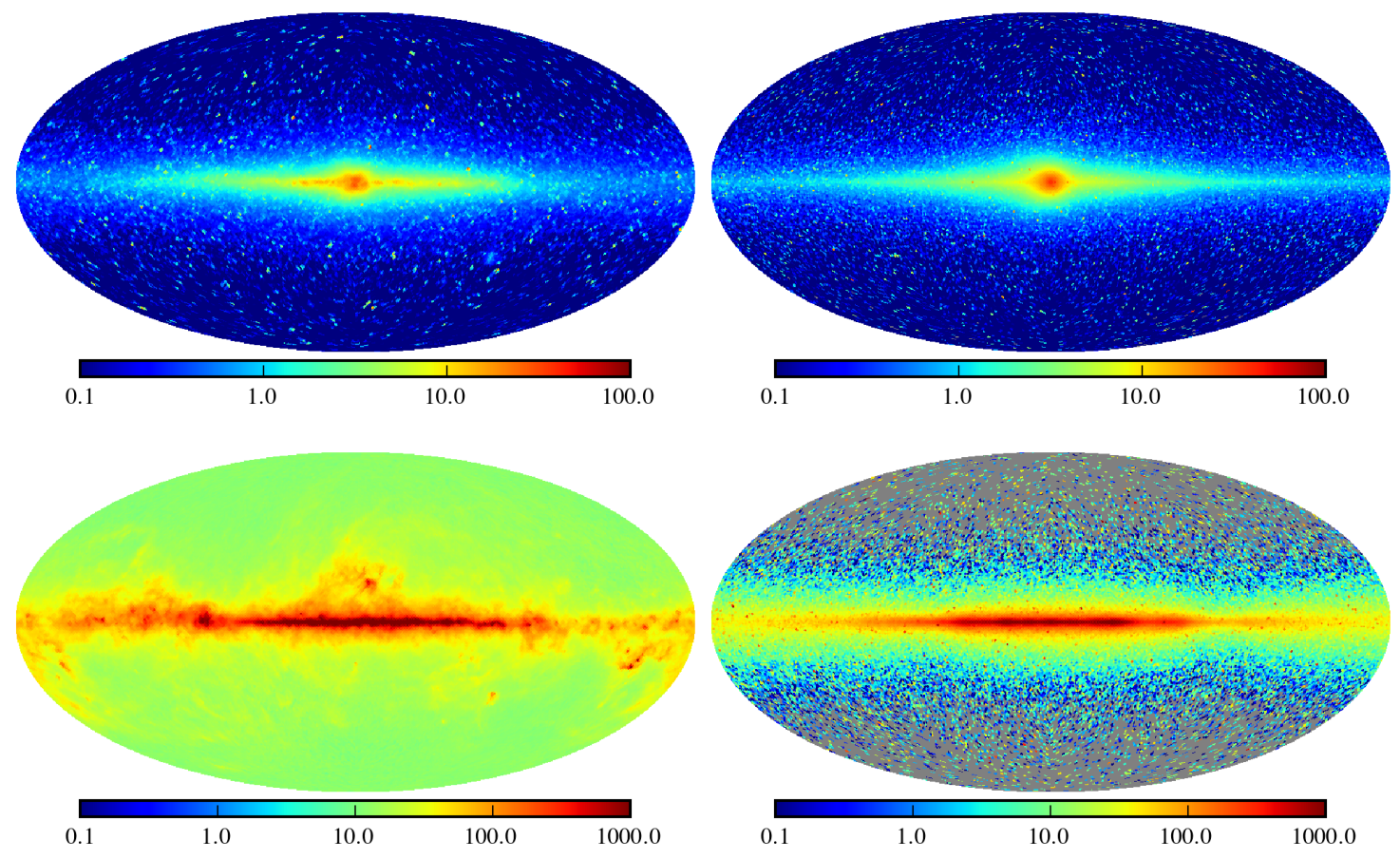

Figure 1: Intensity distribution of near- and far-IR radiation observed at Earth. Left panels show the data from DIRBE band 2 ( $2.2 \mu \mathrm{m}$ central wavelength; upper) and band 9 (140 $\mu \mathrm{m}$ central wavelength; lower) retrieved from NASA HEASARC. Note that source subtraction (point sources, the Magellanic Clouds, etc.) has not been done with these data; in the plane the majority of the emission is due to the interstellar processes and this does not affect the comparison with the model calculations. Right panels show the FRaNKIE code calculations for the model parameters given in the text convolved with the DIRBE instrument response over the same bands shown in the left panels. Overly bright pixels for the FRaNKIE calculations, particularly outside of the Galactic plane, are due to Monte Carlo noise. No smoothing has been applied to the FRaNKIE calculations. The highly structured emission seen in the lower-left panel (DIRBE data for band 9) is absent in the lower-right panel because of the relative simplicity of the dust model that is used for the FRaNKIE calculations (see text). Colour bar units: $\mathrm{MJy} \mathrm{sr}^{-1}$.

with some small morphological differences toward the inner Galaxy where the bulge/bar characterisation is most important. In particular, the bulge appears too bright, while the disk is comparatively less bright than the data indicate. On the other hand, the far-IR filter model prediction is less bright along the Galactic ridge compared to the data. It is also significantly less-rich in the highly structured emission outside of the Galactic plane. This is due to the relative simplicity of the dust model used for the FRaNKIE calculation that is spatially smooth and lacks structure for relatively nearby dust ( $\sim 1 \mathrm{kpc})$, which is seen in the data.

The parameters that have been used for calculating the 2D axisymmetric ISRF do not produce the best visual match when employed in a full 3D calculation. But, there are also additional parameters that have not been adjusted at all, e.g., the bulge/bar offset angle, or the spiral arm parameters. While the 3D models are more realistic they come with the price of a larger number of parameters. The simple tuning 'by eye' that was employed for the 2D ISRF models is inadequate. 


\section{Future Prospects}

There is a wealth of data now available that can be used to improve the modelling of the spatial distribution of stars and dust in the Galaxy. All-sky intensity maps spanning the near- to far-IR are available for DIRBE/COBE (as seen above), with the reprocessed IRAS (IRIS) also covering the mid- to far-IR. Spitzer IRAC data (GLIMPSE-I/II/3D/360) covers most of the Galactic plane from $3.6 \mu \mathrm{m}$ to $8.0 \mu \mathrm{m}$ within a few degrees, but extends out to about $\pm 4^{\circ}$ toward the inner Galaxy (to cover the Galactic bulge). Spitzer MIPS data (MIPSGAL) covers the inner Galactic plane in the mid-IR (only the $24 \mu \mathrm{m}$ data is available currently). Planck intensity data is available for wavelengths into the microwave range.

Synthesising a single model that describes these data is a challenging task. The number of relevant model parameters is potentially large (e.g., disk scale-length, spiral arm geometry, bulge position angle and central luminosity, dust-to-gas ratio spatial variation). As a callable library, the FRaNKIE code can be used to generate synthetic intensity maps for any set of model parameters. We are in the process of combining FRaNKIE with codes such as MULTINEST [38] or custom Markov Chain Monte Carlo to enable statistical evaluation of multi-parametric models for the 3D Milky Way ISRF. This will better inform the uncertainties associated with predictions of the diffuse $\gamma$-ray emission from CR electrons and positrons losing energy via the inverse Compton scattering of the low-energy photons of the MW ISRF. Accurate predictions of the diffuse $\gamma$-rays from this process made using the ISRF with the GALPROP code are essential for understanding the potential "excesses" in the residuals of the high-quality Fermi-LAT data, particularly toward the inner Milky Way [39]. In addition, self-consistent 3D ISRF models are essential for studying the Galactic foregrounds due to dust emission and the properties of the dust grains themselves.

\section{Acknowledgments}

FRANKIE and GALPROP development are supported through NASA Grants NNX10AE78G and NNX13AC47G

\section{References}

[1] T. A. Porter, I. V. Moskalenko, A. W. Strong, E. Orlando, and L. Bouchet, Inverse Compton Origin of the Hard X-Ray and Soft Gamma-Ray Emission from the Galactic Ridge, ApJ 682 (2008) 400-407, [arXiv:0804.1774].

[2] L. Bouchet, A. W. Strong, T. A. Porter, I. V. Moskalenko, E. Jourdain, and J.-P. Roques, Diffuse Emission Measurement with the SPectrometer on INTEGRAL as an Indirect Probe of Cosmic-Ray Electrons and Positrons, ApJ 739 (2011) 29, [arXiv: 1107.0200 ].

[3] M. Ackermann, and Fermi-LAT Collaboration, Fermi-LAT Observations of the Diffuse $\gamma$-Ray Emission: Implications for Cosmic Rays and the Interstellar Medium, ApJ 750 (2012) 3, [arXiv:1202.4039].

[4] T. A. Porter, I. V. Moskalenko, and A. W. Strong, Inverse Compton Emission from Galactic Supernova Remnants: Effect of the Interstellar Radiation Field, ApJL 648 (2006) L29-L32, [astro-ph/0607344]. 
[5] I. V. Moskalenko, T. A. Porter, and A. W. Strong, Attenuation of Very High Energy Gamma Rays by the Milky Way Interstellar Radiation Field, ApJL 640 (2006) L155-L158, [astro-ph/ 051149 ].

[6] A. N. Witt, S. Mandel, P. H. Sell, T. Dixon, and U. P. Vijh, Extended Red Emission in High Galactic Latitude Interstellar Clouds, ApJ 679 (2008) 497-511, [arXiv : 0802 . 0674 ].

[7] A. A. Abdo, and Fermi-LAT Collaboration, Spectrum of the Isotropic Diffuse Gamma-Ray Emission Derived from First-Year Fermi Large Area Telescope Data, Physical Review Letters 104 (2010) 101101, [arXiv:1002.3603].

[8] M. Ackermann, and Fermi-LAT Collaboration, The spectrum of isotropic diffuse gamma-ray emission between $100 \mathrm{MeV}$ and $820 \mathrm{GeV}$, ArXiv e-prints (2014) [arXiv: 1410.3696 ].

[9] J. S. Mathis, P. G. Mezger, and N. Panagia, Interstellar radiation field and dust temperatures in the diffuse interstellar matter and in giant molecular clouds, A\&A 128 (1983) 212-229.

[10] X. Chi and A. W. Wolfendale, The interstellar radiation field: a datum for cosmic ray physics, Journal of Physics G Nuclear Physics 17 (1991) 987-998.

[11] A. W. Strong, I. V. Moskalenko, and O. Reimer, Diffuse Continuum Gamma Rays from the Galaxy, ApJ 537 (2000) 763-784, [astro-ph/9811296].

[12] T. A. Porter and A. W. Strong, A new estimate of the Galactic interstellar radiation field between 0.1um and 1000um, International Cosmic Ray Conference 4 (2005) 77, [a st ro-ph / 0507119 ].

[13] I. V. Moskalenko and A. W. Strong, Anisotropic Inverse Compton Scattering in the Galaxy, ApJ 528 (2000) 357-367, [astro-ph/9811284].

[14] K. D. Gordon, K. A. Misselt, A. N. Witt, and G. C. Clayton, The DIRTY Model. I. Monte Carlo Radiative Transfer through Dust, ApJ 551 (2001) 269-276, [astro-ph/0011575].

[15] S. Bianchi, Dust extinction and emission in a clumpy galactic disk. An application of the radiative transfer code TRADING, A\&A 490 (2008) 461-475, [arXiv : 0807.2946$].$

[16] T. P. Robitaille, E. Churchwell, R. A. Benjamin, B. A. Whitney, K. Wood, B. L. Babler, and M. R. Meade, A self-consistent model of Galactic stellar and dust infrared emission and the abundance of polycyclic aromatic hydrocarbons, A\&A $\mathbf{5 4 5}$ (2012) A39, [arXiv:1208.4606].

[17] L. B. Lucy, Computing radiative equilibria with Monte Carlo techniques, A\&A 344 (Apr., 1999) 282-288.

[18] T. J. Harries, Radiation-hydrodynamical simulations of massive star formation using Monte Carlo radiative transfer - I. Algorithms and numerical methods, MNRAS 448 (2015) 3156-3166, [arXiv:1501.0575].

[19] J. E. Bjorkman and K. Wood, Radiative Equilibrium and Temperature Correction in Monte Carlo Radiation Transfer, ApJ 554 (2001) 615-623, [astro-ph/ 010324 9].

[20] B. T. Draine and A. Li, Infrared Emission from Interstellar Dust. I. Stochastic Heating of Small Grains, ApJ 551 (2001) 807-824, [astro-ph/ 0011318 ].

[21] T. A. Porter and A. E. Vladimirov, Calculation of Stochastic Heating and Emissivity of Cosmic Dust Grains with Optimization for the Intel Many Integrated Core Architecture, ArXiv e-prints (2013) [arXiv:1311.4627].

[22] K. M. Górski, E. Hivon, A. J. Banday, B. D. Wandelt, F. K. Hansen, M. Reinecke, and M. Bartelmann, HEALPix: A Framework for High-Resolution Discretization and Fast Analysis of Data Distributed on the Sphere, ApJ 622 (2005) 759-771, [a stro-ph/ 0409513 ]. 
[23] H. T. Freudenreich, A COBE Model of the Galactic Bar and Disk, ApJ 492 (1998) 495-510, [astro-ph/9707340].

[24] R. Garwood and T. J. Jones, Modeling the Milky Way in the infrared, PASP 99 (1987) 453-460.

[25] R. J. Wainscoat, M. Cohen, K. Volk, H. J. Walker, and D. E. Schwartz, A model of the 8-25 micron point source infrared sky, ApJS 83 (1992) 111-146.

[26] A. J. Pickles, A Stellar Spectral Flux Library: 1150-25000 A, PASP 110 (1998) 863-878.

[27] C. Porcel, F. Garzon, J. Jimenez-Vicente, and E. Battaner, The radial scale length of the Milky Way, A\&A 330 (1998) 136-138, [astro-ph/9710197].

[28] R. Drimmel and D. N. Spergel, Three-dimensional Structure of the Milky Way Disk: The Distribution of Stars and Dust beyond $0.35 R_{\text {solar }}$, ApJ 556 (2001) 181-202, [astro-ph/ 0101259 ].

[29] M. Jurić, and et al., The Milky Way Tomography with SDSS. I. Stellar Number Density Distribution, ApJ 673 (2008) 864-914, [astro-ph/0510520].

[30] J. T. A. de Jong, B. Yanny, H.-W. Rix, A. E. Dolphin, N. F. Martin, and T. C. Beers, Mapping the Stellar Structure of the Milky Way Thick Disk and Halo Using SEGUE Photometry, ApJ 714 (2010) 663-674, [arXiv:0911.3900].

[31] P. J. McMillan, Mass models of the Milky Way, MNRAS 414 (2011) 2446-2457, [arXiv:1102.4340].

[32] J. P. Vallee, Different studies of the global pitch angle of the Milky Way's spiral arms, MNRAS 450 (2015) 4277-4284, [arXiv:1505.0120].

[33] M. López-Corredoira, A. Cabrera-Lavers, and O. E. Gerhard, A boxy bulge in the Milky Way. Inversion of the stellar statistics equation with $2 M A S S$ data, A\&A 439 (2005) 107-110, [astro-ph/0504608].

[34] B. T. Draine and A. Li, Infrared Emission from Interstellar Dust. IV. The Silicate-Graphite-PAH Model in the Post-Spitzer Era, ApJ 657 (2007) 810-837, [astro-ph / 0608003 ].

[35] J. C. Weingartner and B. T. Draine, Dust Grain-Size Distributions and Extinction in the Milky Way, Large Magellanic Cloud, and Small Magellanic Cloud, ApJ 548 (2001) 296-309, [astro-ph/0008146].

[36] R. C. Bohlin, B. D. Savage, and J. F. Drake, A survey of interstellar H I from L-alpha absorption measurements. II, ApJ 224 (1978) 132-142.

[37] A. W. Strong, I. V. Moskalenko, O. Reimer, S. Digel, and R. Diehl, The distribution of cosmic-ray sources in the Galaxy, $\gamma$-rays and the gradient in the CO-to- $\mathrm{H}_{2}$ relation, $A \& A 422$ (2004) L47-L50, [astro-ph/ 0405275$].$

[38] F. Feroz, M. P. Hobson, and M. Bridges, MULTINEST: an efficient and robust Bayesian inference tool for cosmology and particle physics, MNRAS 398 (2009) 1601-1614, [arXiv:0809.3437].

[39] T. A. Porter and S. Murgia, Observations of High-Energy Gamma-Ray Emission Toward the Galactic Centre with the Fermi Large Area Telescope, This conference. 